\title{
OCORRÊNCIA DE EVENTOS EXTREMOS DE PRECIPITAÇÃO EM UBERABA (1959-2015) E SUA RELAÇÃO COM AS MUDANÇAS CLIMÁTICAS
}

\author{
Cléo Maycon Viana Paz ${ }^{(a)}$, Fabio de Oliveira Sanches ${ }^{(b)}$ \\ (a) Bolsista de IC FAPEMIG, Universidade Federal do Triângulo Mineiro, cleomaycon.vianapaz@ hotmail.com \\ (b) Universidade Federal do Triângulo Mineiro, fsanches.73@gmail.com
}

\section{Eixo: Climatologia em Diferentes Níveis Escalares: Mudanças e Variabilidades}

\begin{abstract}
Resumo
Nos últimos anos, os efeitos das mudanças climáticas globais e suas respectivas repercussões tornaram-se temas de diversos encontros científicos, sobretudo após a sequência de relatórios (Assessment Report) divulgados pelo Painel Intergovernamental sobre Mudanças Climáticas (IPCC em inglês). Nesse sentido, o principal objetivo desse trabalho é identificar a ocorrência de eventos intensos de precipitação ao longo dos anos de 1961 a 2015, e o tempo de retorno desses eventos. Foram utilizados dados da Estação Meteorológica de Superfície em Uberaba (OMM: 83577) para o período de 1961-2015. A partir dos parâmetros propostos pelo (ETCCDI), foram identificados e analisados os parâmetros pluviométricos. A análise revelou que as chuvas nos meses de março tornaram-se mais frequentes e intensas, bem como as chuvas intensas em maio. De acordo com os resultados encontrados, os eventos pluviométricos extremos têm se tornado mais frequentes e intensos nos últimos 56 anos em Uberaba, sobretudo nos meses março e maio.
\end{abstract}

Palavras chave: Mudanças Climáticas, chuvas intensas; Triângulo Mineiro.

\section{Introdução}

Nos últimos anos, os efeitos das mudanças climáticas globais e suas respectivas repercussões nos níveis regionais e locais tornaram-se temas de diversos encontros científicos, sobretudo após a sequência de relatórios (Assessment Report) divulgados pelo Painel Intergovernamental sobre Mudanças Climáticas (IPCC em inglês).

É de suma importância estudar tais eventos extremos de precipitação, pois, os mesmos são responsáveis por grandes fenômenos de alagamentos, trazendo perdas econômicas, de vida humana, e, demais transtornos que justificam tamanho interesse científico pela temática.

Nesse sentido, o principal objetivo desse trabalho é identificar a ocorrência de eventos intensos de precipitação ao longo dos anos de 1961 a 2015, e o tempo de retorno desses eventos.

O presente trabalho é resultado de atividades realizadas no projeto de iniciação científica que foi desenvolvida na Universidade Federal do Triângulo Mineiro (UFTM), campus Uberaba/MG, de março de 2016 a fevereiro de 2017. 


\section{Materiais e métodos}

Para desenvolvimento deste trabalho foram utilizados dados pluviométricos da Estação Meteorológica de Superfície de Uberaba (OMM: 83577), obtidos por meio do Instituto Nacional de Meteorologia (INMET), através de seu Banco de Dados Meteorológicos para o Ensino e Pesquisa (BDMEP), o acesso a essa plataforma é obtido pelo site (http://www.inmet.gov.br/portal/index.php?r=bdmep/bdmep).

Todos os procedimentos de preparação dos dados (preenchimento de falhas existentes, métodos e testes estatísticos, análise de consistência, software utilizado etc.) estão descritos em estão Paz et al (2016).

De posse de uma série temporal de 56 anos de dados pluviométricos diários, foram extraídos os parâmetros propostas pelo Expert Team on Climate Change Detection and Indices (ETCCDI) para a detecção evidências de mudanças climáticas.

Através do software Excel os dados foram extraídos os parâmetros $R>70, R>80$ e $R>100$ para respectivamente chuvas a partir de $70 \mathrm{~mm}, 80 \mathrm{~mm}$ e $100 \mathrm{~mm}$, bem como o percentil 99 (valor extremo para a pluviosidade diária), os valores pluviométricos diários foram segmentados até o valor máximo observado.

Para cada um dos parâmetros extraídos foi aplicado o Teste de Mann-Kendall (MK), para qualificar os dados como passíveis ou não de mudança de comportamento ao longo da série. Neste teste, adotar-se a hipótese da estabilidade da série temporal $\left(H_{0}\right)$ onde os valores devem ser independentes e a distribuição de probabilidades deve permanecer sempre a mesma.

O MK é um teste não paramétrico utilizado para determinar se determinada série de dados possui uma tendência temporal de alteração estatisticamente significativa.

Uma vez que apenas a avaliação e verificação dos parâmetros como tendência negativa ou positiva não é suficiente para qualificar tal alteração como mudança de comportamento, coube a aplicação do Teste MK para avaliação com nível de confiança de $95 \%$ ( $p$-value: 0,05). Para isso, o valor do score z deve ser -1,96 $\leq \mathrm{z} \leq 1,96$, conforme recomenda Marengo e Alves (2005) e Folhes e Fisch (2006).

O MK tem sido empregado em diversos trabalhos como objetivo de encontrar evidências de mudanças de comportamento que possam ser consideradas como evidências de mudanças climáticas (PAIVA e CLARKE, 1995; GROPPO et al., 2001; MARENGO e ALVES, 2005; ALEXANDER et al., 2006; FOLHES e FISCH, 2006; OBREGÓN e MARENGO, 2007; BLAIN, 2010; SANCHES et al., 2014; PAZ et al.,2016.)

Em um segundo momento, foi extraído o percentil 99 dos dados pluviométricos diários. O objetivo de se extrair o percentil 99 foi o de identificar o valor referente às chuvas extremas diárias da série estudada. 
A partir do valor do percentil 99 (valor extremo para a pluviosidade diária), os valores pluviométricos diários foram segmentados até o valor máximo observado. Foi identificado o valor de $52,4 \mathrm{~mm} \cdot$ dia $^{-1}$ como valor percentil 99.

A segmentação considerou os eventos pluviométricos nos intervalos entre $52,4 \mathrm{~mm}$ e $60 \mathrm{~mm}, 60 \mathrm{~mm}$ e 80 $\mathrm{mm}, 80 \mathrm{~mm}$ e $100 \mathrm{~mm}, 100 \mathrm{~mm}$ e $120 \mathrm{~mm}$ e acima de $120 \mathrm{~mm}$.

A partir desses valores, foi extraída a frequência absoluta $(f)$ dos eventos, que corresponde a quantidade de eventos ocorridos no período; a frequência relativa $(f r)$ que corresponde ao número de vezes que o evento ocorreu (na) em relação ao número total de elementos da série $(n)$ e o seu Tempo de retorno $(T)$ ao longo dos 56 anos da série.

A partir dessas informações foi possível estabelecer o comportamento dos dados em relação a sua ocorrência identificando se tais eventos estão se tornando ou não mais frequentes e intensos.

\section{Resultados e Discussões}

Ocorreram quatro eventos com pluviosidade acima de $120 \mathrm{~mm}$ ao longo da série, tendo seu tempo de retorno de 14 anos (Tabela I);

Ocorreram oito eventos com pluviosidade entre 100 e $120 \mathrm{~mm}$ ao longo da série, com tempo de retorno de 14 anos (Tabela I);

Ocorreram 28 eventos com pluviosidade entre 80 e $100 \mathrm{~mm}$, com tempo de retorno de dois anos (Tabela I);

Ocorreram 92 eventos de com pluviosidade entre 60 e $80 \mathrm{~mm}$, com seu tempo de retorno de 0,6 anos (Tabela I); Ocorreram 78 eventos com pluviosidade entre 62,4 e $60 \mathrm{~mm}$, com tempo de retorno de 0,7 anos (Tabela I).

Tabela I - Tempo de retorno (tempo de recorrência em anos)

\begin{tabular}{|c|c|c|c|}
\hline Eventos & Frequêcia Absoluta & Frequêcia Relativa & Tempo de retorno em anos \\
\hline$>120 \mathrm{~mm}$ & 4 & 0,07142857 & 14 \\
\hline $100-120 \mathrm{~mm}$ & 8 & 0,14285714 & 7 \\
\hline $80-100 \mathrm{~mm}$ & 28 & 0,50000000 & 2 \\
\hline $60-80 \mathrm{~mm}$ & 92 & 1,64285714 & 0,6 \\
\hline $52,4-60 \mathrm{~mm}$ & 78 & 1,39285714 & 0,7 \\
\hline
\end{tabular}




\section{Conclusões}

De acordo com os resultados encontrados, os eventos pluviométricos extremos tem se tornado mais frequentes e intensos nos últimos 56 anos em Uberaba. Há um aumento na quantidade e na intensidade das chuvas nos meses de março. Eventos extremos de precipitação também têm ocorrido com maior frequência nos meses de maio.

Já, no mês de outubro, o fenômeno é outro. O aumento na quantidade de dias secos e, consequentemente, a redução significativa das chuvas está prolongando a estação seca, retardando o retorno da estação chuvosa.

\section{Bibliografia}

ALEXANDER, L.V; ZHANG, X.; PETERSON, T.C.; CAESAR, J.; GLEASON, B.; KLEIN TANK, A.M.G.; HAYLOCK, M.; COLLINS, D.; TREWIN, B.; RAHIMZADEH, F.; TAGIPOUR, A.; RUPA KUMAR, K.; REVADEKAR, J.; GRIFFITHS, G.; VICENT, L.; STEPHENSON, D. B.; BURN, J.; AGUILAR, E.; TAYLOR, M.; NEW, M.; ZHAIN, P.; RUSTICUCCI, M.; VAZQUEZ-AGUIRRE, J.L. Global observed changes in daily climate extremes of temperature and precipitation. Journal of Geophysical Research. vol. 111, D05109, 2006. (doi: 10.1029/2005JD00690).

BLAIN, G.C. Detecção de tendências monótonas em séries mensais de precipitação pluvial no Estado de São Paulo. Bragantia, Campinas, v.69, n4, p.1027-1033, 2010.

FOLHES, M.T.; FISCH, G. Caracterização climática e estudo de tendência nas séries temporais de temperatura do ar e precipitação em Taubaté (SP). Ambi-Agua, Taubaté, v.1, n.1, p.61-71, 2006.

GROPPO, J.D.; MILDE, L.C.E.; GUAMERO, M.E.; MORAES, J.M.; MARTINELLI, L.A. Análise de séries temporais de vazão e de precipitação na Bacia do Rio Piracicaba. Revista de Ciência \& Tecnologia. v.8, n.18, p.109-117, 2001.MARENGO, J.A.; ALVES, L.M. Tendências hidrológicas da bacia do rio Paraíba do Sul. Revista Brasileira de Meteorologia, v.20, n.2, 215-226, 2005.

OBREGÓN, G.; MARENGO, J.A. Caracterização do clima no Século XX no Brasil: Tendências de chuvas e Temperaturas Médias Extremas. Relatório $\mathrm{n}^{\circ}$ 2. Ministério do Meio Ambiente. Secretaria de Biodiversidade e Florestas. Diretoria de Conservação da Biodiversidade. 2007.

PAIVA, E.M.C.D.; CLARKE, R.T. Análise de tendência de precipitação na Amazônia. Revista Brasileira de Meteorologia. n.10 (1/2), p.37-41, 1995.

PAZ, C.M.; SANCHES, F.O.; FERREIRA, R.V.; MACHADO, V. Tendências Pluviométricas Das Chuvas Para Uberaba (1961-2015). In: XII Simpósio Brasileiro de Climatologia Geográfica. Anais... Goiânia-GO. 2016.

SANCHES, F.O.; VERDUM, R.; FISCH, G. Tendência de longo prazo das chuvas diárias no Sudoeste do Rio Grande do Sul: os eventos extremos e a arenização. Revista Brasileira de Geografia Física, v. 7, p. 1100-1109, 2014. 